\title{
Assessing the Progress of Implementing Web Accessibility: An Irish Case Study
}

\author{
Vivienne Trulock \\ Ilikecake Limited, 12 Lealand Road, Clondalkin, Dublin 22, Ireland \\ vivienne@ilikecake.net \\ Richard Hetherington \\ Centre for Interaction Design, School of Computing, Napier University, 10 Colinton Road, Edinburgh EH10 5DT, UK \\ r.hetherington@napier.ac.uk
}

Keywords: Web Accessibility, WCAG Guidelines 1.0, automated testing, manual testing, partial accessibility.

\begin{abstract}
In this paper we attempt to gauge the implementation of web accessibility guidelines in a range of Irish websites by undertaking a follow-up study in 2005 to one conducted by McMullin three years earlier (McMullin, 2002). Automatic testing against version 1.0 of the Web Content Accessibility Guidelines (WCAG 1.0) using WebXact online revealed that accessibility levels had increased among the 152 sites sampled over the three-year period. Compliancy levels of A, AA and AAA had risen from the 2002 levels of $6.3 \%, 0 \%$ and $0 \%$ respectively to $36.2 \%, 8.6 \%$ and $3.3 \%$ in 2005 . However, manual checks on the same sites indicated that the actual compliance levels for 2005 were $1.3 \%, 0 \%$ and $0 \%$ for A, AA and AAA. Of the sites claiming accessibility, either by displaying a W3C or 'Bobby' compliance logo, or in text on their accessibility statement page, $60 \%$ claimed a higher level than the automatic testing results indicated. When these sites were further manually checked it was found that all of them claimed a higher level of accessibility compliance than was actually the case. As most sites in the sample were not compliant with the WCAG 1.0 for the entire set of disabilities, the concept of 'partial accessibility' was examined by identifying those websites that complied with subsets of the guidelines particular to different disabilities. Some disability types fared worse than others. In particular blindness, mobility impairment and cognitive impairment each had full support from at most $1 \%$ of the websites in the study. Other disabilities were better supported, including partially-sighted, deaf and hearing impaired, and colour blind, where compliance was found in $11 \%, 23 \%$ and $32 \%$ of the websites, respectively.
\end{abstract}

\section{INTRODUCTION}

The importance of access to the World Wide Web cannot be underestimated. This is particularly so for those individuals who are disabled in such a way as to render access to traditional media difficult to attain or to use effectively. Within the last decade, many countries have begun to implement a legal requirement for websites to be accessible. Often this has been the result of general disability or equality legislation, rather than legislation directed specifically at online access. In Ireland for example, Part 1 Section 4(1) of the Equal Status Act, Ireland,
2000 states that a failure to do all that is reasonable to provide a service to a person with a disability is deemed an act of discrimination (Irish Government, 2000). The Employment Equality Act of Ireland, 1998, Section 16(3) (Irish Government, 1998) has a similar definition. Whilst The Disability Act, Ireland, 2005 states in section 27(1) that the head of the organisation is responsible for ensuring that services are available to people with disabilities (Irish Government, 2005). A website then, if regarded as a service, must be as available to a disabled person as it is to an able bodied person otherwise the service is discriminatory. Available redress includes compensation and an order that the problem(s) be fixed or removed (Irish Government, 
2000). At present, no cases regarding website access have been pursued under these Acts.

The European Union, of which Ireland is a member, has been proactive in developing explicit web accessibility guidelines. The eEurope 2002 Action Plan states that the content of public sector web sites in Member States and in European Institutions must be designed to be accessible to ensure that citizens with disabilities can access information and take full advantage of the potential for e-government (European Commission \& Council, 2000). The timeframe for adoption of the Web Accessibility Initiative (WAI) guidelines by public websites was designated to be the end of 2001. A separate communication from the EU, 'eEurope 2002: Accessibility of Public Web Sites and their Content', recognised the WAI WCAG 1.0 guidelines to be the 'global de facto Web accessibility standard' and concluded that both public and private websites should be encouraged to achieve accessibility during 2003, the European Year of Disabled People (European Commission, 2001).

Considering the significant introduction of legislation addressing online accessibility, either directly or indirectly, over the last 10 years, an investigation of the impact of legislation and associated guidelines on the accessibility of web sites appears timely, in order to assess just how much, or how little progress is being made. However, in order to establish where we are in terms of accessibility, we need to know where we've been. In the Irish context we are fortunate in having access to a study that determined the accessibility of a sample of Irish web sites in 2002 (McMullin, 2002). Using these data as the baseline, a follow-up study on the same sites was undertaken to re-assess their accessibility and compliance levels to WCAG 1.0 in 2005. In this paper we report our major findings.

\section{METHODOLOGY}

\subsection{Guidelines}

Websites were assessed for accessibility using WCAG version 1.0 (W3C, 1999). These guidelines are an 'indicator of web accessibility' (McMullin, 2002) and consist of 14 separate guidelines and 65 specific checkpoints, which are broken into 3 levels of priority: priority $1,2 \& 3$. Priority 1 guidelines must be met in order to afford basic accessibility. Priority 2 guidelines should be met to offer additional access to a broader range of disabled groups. Priority 3 guidelines may be met to provide further additional support (Brewer, 2004; McMullin,
2002; Williams \& Rattray, 2003; Sullivan \& Matson, 2000; Hackett, Parmanto \& Zeng, 2004).

There are 3 levels of compliance with the WCAG 1.0 guidelines: A, AA and AAA. The compliance level of $\mathrm{A}$ means that all priority 1 guidelines are satisfied. The compliance level of AA means that all priority 1 and 2 guidelines are satisfied. AA is considered to be 'professional standard'. The compliance level of AAA means that all priority 1, 2 and 3 guidelines are satisfied. AAA is considered to be 'gold standard' (Brewer, 2004; McMullin, 2002; Loiacono \& McCoy, 2004; Hackett, et al, 2004). Note that in order for a site to be truly compliant to any particular level it must satisfy all the checkpoints to that level, not simply those which can be verified by accessibility verification software.

\subsection{Accessibility Testing}

The 159 site URLs from McMullin's 2002 study (McMullin, 2002) were used to retrieve websites for testing and analysis. Of these, three websites had placeholder pages and four sites were not available as the URL had not been renewed. Consequently, the total number of websites analysed in the current study was 152 . Of these, 101 sites had the original URL used in the 2002 study, 40 had an automatic redirect to an updated URL and one had a nonautomatic, linked redirect. A further 10 had URLs which were replaced by manual searches in Google, WHOIS and the Enterprise Ireland website. The sample tested represented a considerable range of websites including those belonging to the military, political parties and charities, national and local governments, and public and private commercial sites ranging from large multinationals to smaller local companies.

In the present study, the home or index page was checked in greatest detail. The home page is generally the point at which most users access a web site. Therefore, if a home page is inaccessible, there may be no way for a disabled user to access the rest of the site (Sullivan \& Matson, 2000). In addition, the home page of a web site tends to be the page that is the best planned and coordinated, unlike lowerlevel content pages which can be managed by different departments or individuals. Therefore, it is likely that if any web pages are accessible, the home page is. (Lazar, Beere, Greenidge \& Nagappa, 2003). Moreover, the entry page can be taken as a good signifier of a web site's overall accessibility level (Williams \& Rattray, 2003). However, in order to ascertain a true measure of compliance, manual and automatic checks were performed on the other pages of a website. As some manual checks cannot 
reasonably be performed without user simulation, this was included where appropriate in the testing.

Some of the sites in this study used framesets or iframes as part of their design. As the automatic validator only analyzes the URL submitted and not the embedded frame pages, these were analyzed separately. Therefore a page using frames is deemed to have an accessibility rating equal to that of the frameset plus that of each of the pages viewed in the frameset on page load. Pages using iframes also had the accessibility results of the iframe page added to the original page.

The WebXact validator available at http://webxact.watchfire.com/ was chosen as the automated testing tool. The 2002 study used Bobby, however WebXact replaced Bobby on-line just prior to this study and the Bobby URL (http://bobby.watchfire.com/) redirected to WebXact.

Initial analysis concentrated on the automatically verifiable checkpoints allowing for a direct comparison to be made between the 2002 and the 2005 results. Checkpoints not failing the automated test were recorded as passing the validation. Where appropriate (e.g. when directed by the automatic testing tool), manual checks were undertaken and an additional analysis carried out. In the cases where checkpoints validated both manually and automatically, the checkpoint was considered to have been passed. A checkpoint was deemed to have been failed if either manual or automatic testing revealed a failure.

The complete method for performing manual checks of web pages has been described (Trulock, 2006) and involved the use of additional software tools to validate specific checkpoints: The JAWS 6.20 screen reader was used to determine if accessible text versions or alternative text descriptions where applied to any time-based multimedia present on web pages (checkpoint 1.4). Colour contrast between foreground and background (checkpoint 2.2) was checked using an accessibility tool called 'aDesigner' (Takagi, Asakawa, Fukuda \& Maeda, 2004). The default settings were used, which simulated a crystalline lens transparency of 40 years old, in addition to 3 types of colour blindness. Web pages had to pass all 4 conditions to achieve validation.

Compliance of documents with formal grammar specifications (checkpoint 3.2) was checked at http://validator.w3.org/ for html and xhtml. Cascading Style Sheets (CSS ) were validated at http://jigsaw.w3.org/css-validator/validator-uri

(CSS1, CSS2). Where xhtml files fail the check and the associated CSS files cannot be assessed, the CSS file was tested separately at http://jigsaw.w3.org/cssvalidator/ using the 'validate by file upload' option.
Pages which failed any applicable test were deemed to have failed the check. Browser settings were adjusted in order to test whether documents could be read without style sheets (checkpoint 5.2) and also to ensure that pages were usable when scripts, applets or other programmatic objects were turned off (checkpoints 6.1 and 6.3).

A flickering check tool available at http://www.webaccessibile.org/test/check.aspx was used to check for flickering animated gifs, which are covered by checkpoint 7.1. Flickering elements outside the critical range (4-59 flashes/second) were deemed to have passed (W3C, 1999).

In order to assess the readability of text, a testing tool available from Juicy Studios was used (http://juicystudio.com/services/readability.php).

Pages that obtained a Flesch-Kincaid Grade Level of $5^{\text {th }}$ Grade (5.x) or lower where considered to have satisfied the related checkpoint (checkpoint 14.1).

Finally, the correct linearisation of tables (checkpoint 5.3) was checked by viewing with the Lynx text-only viewer (http://lynx.isc.org/current/). Lynx treats the $\langle$ tr $>$ tag as a $\langle$ br $>$ tag, and the $\langle$ tr $>$ and $\langle\mathrm{td}\rangle$ tags as spaces, effectively linearising a table.

\section{RESULTS AND DISCUSSION}

\subsection{Comparison of 2002 and 2005 Accessibility Levels}

In 2002, an accessibility study of 159 Irish websites revealed that around 6 percent of websites checked were accessible to the minimum level of accessibility, level A (McMullin, 2002). The study only checked automatically verifiable checkpoints and no sites were compliant to Level AA, or Level AAA. Table 1 shows the level of compliance of 152 of these sites tested in 2005 using both automated checking and a combination of automated and manual checking. Comparing sites using automatic validation only, indicates that there has been an almost 6-fold increase in sites achieving Level A compliance over the three year period with around $36 \%$ of those sites tested now achieving a basic level of accessibility. This suggests that there has been an increase in awareness of accessibility issues and at least some attempt to implement a degree of accessibility over this time. In addition, the proportion of websites achieving higher levels of accessibility compliance also increased from 0 in 2002 (McMullin, 2002) to $8.6 \%$ and $3.3 \%$ for levels $\mathrm{AA}$ and AAA respectively.

While the levels of accessibility of sites in 2005 were dramatically increased compared to their 2002 
levels as determined by automatic checking a different picture emerged when accessibility was determined by automatic checking supplemented by manual checking (Table 1). In fact, the trends previously noted over the three-year period were just the opposite. Only $1.3 \%$ of websites achieved compliance at level A equating to 2 sites out of the 152 checked in 2005. No site reached full compliance for Levels AA and AAA. This result may imply that while web designers are aware of web accessibility they are only ensuring validation of the automatically checked checkpoints and appear to be ignoring those checkpoints that can only be satisfied through additional manual testing.

Table 1: Percentages of a sample of 152 Irish websites found to be accessible in 2005 according to WCAG 1.0 Compliance Levels. Accessibility was determined using both automated and manual testing

\begin{tabular}{|l|r|r|c|}
\hline $\begin{array}{l}\text { Testing } \\
\text { Method }\end{array}$ & $\begin{array}{c}\text { WCAG } \\
\text { Level A }\end{array}$ & $\begin{array}{c}\text { WCAG } \\
\text { Level AA }\end{array}$ & $\begin{array}{c}\text { WCAG } \\
\text { Level AAA }\end{array}$ \\
\hline $\begin{array}{l}\text { Automatic } \\
\text { Check } \\
\text { Only }\end{array}$ & $36.2 \%$ & $8.6 \%$ & $3.3 \%$ \\
\hline $\begin{array}{l}\text { Automatic } \\
\text { and } \\
\text { Manual } \\
\text { Check }\end{array}$ & $1.3 \%$ & $0 \%$ & $0 \%$ \\
\hline
\end{tabular}

\subsection{Accessibility Claims}

In the current study, 20 websites claimed to be compliant to the WCAG 1.0 guidelines either by displaying a W3C or 'Bobby' compliance logo, or in text on their accessibility statement page. By automatic checking alone, seven sites were compliant to the level claimed, 12 sites claimed a higher compliance level than their test results indicated, and one site claimed a lower compliance level. A further 35 sites were compliant to the automatic checks at varying levels but no claim of that compliance could be found on their sites. When a combination of automatic and manual checking was carried out, all 20 sites were found to have claimed a higher level of accessibility than the test results indicated. Two sites were identified as being fully compliant to level A, however, one site did not claim any compliance level and the other site claimed compliance of AA.

\section{3 "Partial Accessibility" levels}

Most sites in our sample of websites failed to achieve even basic compliance of WCAG 1.0. However, it is possible that websites may be fully accessible to certain disability groups even though they are not fully compliant. This concept of "partial accessibility' can be assessed by analysing which websites comply with particular subsets of guideline checkpoints. Six disabilities were identified for analysis: fully blind, partially sighted, colour blind, deaf \& hearing impaired, mobility impaired and cognitively impaired. What follows is a general overview of those checkpoints identified as relevant to a specific disability. The sets of checkpoints for each category of disability were evaluated by a combination of both automated and manual checking as described previously. A complete list of all checkpoints identified as relevant to each of the six disabilities can be found in Trulock, 2006.

Of the 65 possible checkpoints, 55 were identified as being relevant to blind individuals. These included text equivalents, appropriate mark up, valid documents, table formatting, device independence and skip links. When these checkpoints were examined, no site passed all of these checkpoints (Table 2).

Six checkpoints were identified for partially sighted users which included text equivalents, good contrast, use of style sheets, use of relative units, and lack of movement on pages. Seventeen sites (11\%) complied with these 6 checkpoints (Table 2).

Four checkpoints were examined which were considered to be relevant to colour blind individuals. These include non-colour formatting, colour contrast and use of style sheets. 48 sites (32\%) were found to be compliant with these 4 checkpoints (Table 2).

Table 2: Number of websites found accessible to specific disabilities for a sample of 152 Irish Websites.

\begin{tabular}{|c|c|}
\hline Disability & $\begin{array}{c}\text { No. of websites } \\
\text { accessible }\end{array}$ \\
\hline Blind & 0 \\
\hline Partially-sighted & 17 \\
\hline Colour blind & 48 \\
\hline Deaf & 35 \\
\hline Mobility impaired & 2 \\
\hline Cognitively impaired & 0 \\
\hline
\end{tabular}

Deaf and hearing impairment were combined as they both require similar treatments in terms of accessible design. Four checkpoints were identified as being relevant to deaf and hearing-impaired individuals, including use of captions, dynamic content equivalents, and clear and simple language. 35 sites (23\%) complied with these 4 checkpoints (Table 2). It should also be noted that most sites checked did not have any specific audio or video content. Clear and simple language is regarded as an important checkpoint because deaf individuals are likely to 
have lower reading levels due to unfamiliarity with the language (Gallaudet Research Institue, 2003).

Checkpoints relating to the mobility impaired numbered 12 and these related to issues including use of relative units, device independence, avoidance of movement, skip links, labels, and sitemaps. Only 2 sites $(1 \%)$ of the sample were compliant with all of these checks (Table 2).

Twenty-two checkpoints relating to cognitive impairment were identified, including text equivalents and supplements, document and navigation structure, no flickering, blinking or moving content, language levels, link targets and alternative search functions. No sites in the sample complied with all of these checkpoints (Table 2).

\subsection{Checkpoint Compliance}

As WCAG 1.0 checkpoint failure rates for automatically verifiable checkpoints were published in the 2002 study ((McMullin, 2002)), a direct comparison of these specific checkpoints can be made with the data obtained in this study. Overall, 14 checkpoints were complied with more often and 6 checkpoints less often in 2005 (Table 3).

Table 3. Percentages of a sample of Irish websites failing to comply with automatically verifiable WCAG 1.0 checkpoints in 2002 and 2005. The 2002 data were obtained from McMullin, 2002.

\begin{tabular}{|c|c|c|c|}
\hline $\begin{array}{c}\text { WCAG 1.0 } \\
\text { Checkpoint }\end{array}$ & $\begin{array}{c}\text { \%Failure } \\
2002\end{array}$ & $\begin{array}{c}\text { \%Failure } \\
2005\end{array}$ & Difference \\
\hline 1.1 & 91.6 & 62 & +29.6 \\
\hline 12.1 & 34.0 & 10 & +24.0 \\
\hline 6.2 & 33.3 & 2 & +31.3 \\
\hline 3.4 & 98.7 & 81 & +17.7 \\
\hline 3.2 & 89.9 & 94 & -4.1 \\
\hline 13.1 & 76.7 & 80 & -3.3 \\
\hline 12.4 & 69.8 & 47 & +22.8 \\
\hline 9.3 & 69.2 & 55 & +14.2 \\
\hline 13.2 & 12.6 & 4 & +8.6 \\
\hline 3.5 & 6.3 & 16 & -9.7 \\
\hline 7.4 & 3.8 & 1 & +2.8 \\
\hline 6.5 & 3.8 & 1 & +2.8 \\
\hline 7.5 & 2.5 & 0 & +2.5 \\
\hline 7.3 & 1.9 & 2 & -0.1 \\
\hline 7.2 & 1.3 & 3 & -1.7 \\
\hline 5.5 & 97.5 & 84 & +13.5 \\
\hline 4.3 & 96.2 & 72 & +24.2 \\
\hline 10.5 & 89.9 & 77 & +12.9 \\
\hline 10.4 & 61.6 & 43 & +18.6 \\
\hline 1.5 & 1.9 & 13 & -11.1 \\
\hline & & & \\
\hline
\end{tabular}

Of the 14 checkpoints that were complied with more often, there was a striking improvement of around $20-30 \%$ of more sites complying for five. These checkpoints related to: providing a text equivalent for every non-text element (1.1), identifying the primary natural language of the document (4.3), ensuring that equivalents for dynamic content are updated when the dynamic content changes (6.2), titling each frame to facilitate frame identification and navigation where framesets are used (12.1) and associating labels explicitly with their controls (12.4). Of the six checkpoints showing a relative reduction in compliance over the threeyear period, five were considered relatively minor having a reduction of around $4 \%$ of sites surveyed or less over the three-year study period. However, for checkpoint 1.5 a reduction in compliance of around $11 \%$ was observed (Table 3 ). This checkpoint relates to client-side image maps requiring alternative text links on them

\section{CONCLUSIONS}

Achieving accessibility to any level is not an easy task. It requires, on the part of the developer: awareness, education, training, organisation, diligence, perseverance, communication and persistence. For the organisations involved it requires time, money, interest, understanding and compromise. For the countries involved it requires public awareness funding, legal consequences for inaction and the belief that disabled individuals have rights to information equal to that of other citizens. While there has been an increase in accessibility levels and awareness of the issue much still needs to be done. The results from this study suggest some effort has been made to achieve a basic level of accessibility compliance as determined by automated checking. Indeed it can be argued that the availability of automated testing tools has made the greatest contribution to the improvement in accessibility levels of the websites sampled. However, such tools have their limitations (Trulock, 2006), and a combination of automated and manual checking, and conducting an evaluation of partial accessibility for specific disabilities revealed that sadly, the majority of sites examined in this study are still excluding many users.

There are several iterative steps involved in implementing online accessibility under the current guidelines. First of all, there needs to be an awareness of the accessibility issue, in that there is an issue. Education of web developers and promotion of accessibility issues will raise the profile of the accessibility movement. This should include the updating of all web modules currently taught in colleges and universities to include 
accessibility issues. In addition, public awareness of accessibility and equality mandates and laws should also increase the likelihood that a client will request an accessible website during the initial consultation phase. This can be accomplished through general advertising in the media, or delivered during seminars to public interest groups.

Web developers need to understand how to actually implement a site that conforms to their relevant guidelines whether they are Section 508 (USA), WCAG 1.0 (EU and Australia) or the Common Look and Feel Guidelines (Canada). In many countries WCAG 1.0 has been widely regarded as the standard for web accessibility. The release of WCAG 2.0 is imminent, and promises a series of guidelines and principles, which will be more precisely testable and more relevant to the advanced technologies now found on the web (W3C, 2008). It will be interesting to monitor the effect of WCAG 2.0 on levels of web accessibility.

Considering the wide range of expertise possessed by individuals tasked with authoring web pages, implementing an accessible web site is far from trivial. Additional training on the part of the developer may be required, which could be selfdirected or formalised in seminars, and should include both understanding of web accessibility issues and specific practical skills development on how these guidelines should be implemented. One third of the sites surveyed here were at least partially compliant, but more should be done regarding raising education levels of designers.

Websites should be created with accessibility standards in mind. An accessibility statement should be created as part of the design guidelines to ensure that standards are adhered to both during the initial design phase and during subsequent site updates. This statement should include the level of accessibility to which the site is being designed. The site should then be tested for conformity to the guidelines. Several automatic checking systems are available. These are a good place to start, however, all the manual checks should also be checked and passed by the designer. This can be difficult as some guidelines can easily be misinterpreted. In response to this situation, a resource website, hosted at http://www.accessibleireland.net has been created by the first author in an attempt to clarify and elaborate upon some of these issues. Also, it may help developers to join a mailing list or network of like minded individuals. In Europe/Ireland organizations include: IRL-DeAN (Irish Design-for-all eAccessibility Network), E-DeAN (European Design for All e-Accessibility Network), IDD (Institute for Design \& Disability), EIDD (European Institute for Design \& Disability) and GAWDS (Guild of Accessible Web Designers).
Websites should also be retested regularly for compliance. In some cases, changes made to the site can themselves be non-compliant making it necessary to retest the site after changes are made. Ideally, the site should be evaluated by actual users, both disabled and otherwise, on a variety of platforms, systems, resolutions, text sizes and colour availability. This is necessary to ensure that the site is actually usable. A study by the Disability Rights Commission claimed that up to $45 \%$ of the problems experienced by disabled users were not a violation of any WGAC 1.0 Checkpoint, and would not have been detected without user testing (Disability Rights Commission, 2004). Again, changes made may cause further confusion to other groups of users, so all changes must be retested and re-evaluated by appropriate users to ensure that changes are effective and acceptable. Subsequent to user testing it is also necessary to retest the web site for conformance to the guidelines, as changes made during the user testing updates may themselves be non-compliant. This testing should also be done on normal site updates before they are posted live. Finally, a feedback form should be included with the web site in the event that unforeseen problems arise for some users. The feedback should be checked regularly and any required changes made as soon as possible.

\section{REFERENCES}

Brewer, J., 2004 Web Accessibility Highlights and Trends. Proceedings of W4A at WWW2004, May 18. 51-55. Retrieved December 132004 from http://portal.acm.org

Disability Rights Commission, 2004. The Web: Access and Inclusion for Disabled People, London. Retrieved June 30, 2005 from http://www.drcgb.org/publicationsandreports/2.pdf

European Commission, 2001. eEurope 2002: Accessibility of Public Web Sites and their Content. Retrieved June 282005 from http://europa.eu.int/eurlex/en/com/cnc/2001/com2001_0529en01.pdf

European Commission \& Council. 2000. An Information Society For All: Action Plan. Retrieved June 28, 2005 from http://europa.eu.int/information_society/eeurope/2002/ action_plan/pdf/actionplan_en.pdf

Gallaudet Research Institute, 2003. Literacy \& Deaf Students Retrieved April 202006 from http://gri.gallaudet.edu/Literacy/\#reading

Hackett, S., Parmanto, B. \& Zeng, W., 2004. Accessibility of Internet Websites through Time. Proceedings of ASSEST'04, October 18-20. 32-39. Retrieved March 9 2005 from http://portal.acm.org 
Irish Government, 1998. Employment Equality Act. Retrieved March 302006 from http://www.gov.ie/bills28/acts/1998/a2198.pdf

Irish Government, 2000. Equal Status Act. Retrieved 28 June 2005 from http://www.oireachtas.ie/documents/bills28/acts/2000/ a800.pdf

Irish Government, 2005. Disability Act. Retrieved 30 March 2006 from http://www.oireachtas.ie/documents/bills28/acts/2005/ a1405.pdf

Lazar. J., Beere, P., Greenidge, K. \& Nagappa, Y., 2003. Web Accessibility in the Mid-Atlantic United States: A Study of 50 Home Pages. Retrieved June 162005 from http://triton.towson.edu/ jlazar/web_accessibility_in_ us.pdf

Loiacono, E. \& McCoy, S., 2004. Web site accessibility: an online sector analysis. Information Technology \& People, 17(1), 87-101.Retrieved March 102005 from http://www.emeraldinsight.com/0959-3845.htm

McMullin, B., 2002. WARP: Web Accessibility Reporting Project Ireland 2002 Baseline Study. 1-82. Retrieved March 152005 from http://eaccess.rince.ie/whitepapers/2002/warp-2002-00/warp-2002-00.pdf

Sullivan, T. \& Matson R., 2000. Barriers to USE: Usability and Content Accessibility on the Webs Most Popular Sites. Proceedings of the Conference of Universal Usability. Arlington VA: ACM, 139-144. Retrieved December 132004 from http://portal.acm.org

Takagi, H., Asakawa, C., Fukuda, K. \& Maeda, J. (2004). Accessibility Designer: Visualizing Usability for the Blind. ACM SIGACCESS Accessibility and Computing (77-78) 177-184. Retrieved March 9, 2005 from http://portal.acm.org

Trulock, V., 2006. A Comparative Analysis of Accessibility Levels of Irish Websites. MSc Dissertation, Napier University.

W3C, 1999. Web Content Accessibility Guidelines. Retrieved June 152005 from http://www.w3.org/TR/WAI-WEBCONTENT

W3C, 2008. Web Content Accessibility Guidelines 2.0. W3C Working Draft 11 December 2007. Retrieved February 282008 from http://www.w3.org/TR/WCAG20/

Williams, R. \& Rattray, R., 2003. An assessment of web accessibility of UK accountancy firms. Managerial Auditing Journal, 9(18), 710-716. Retrieved March 10 2005 from http://www.emeraldinsight.com/02686902.htm 